\title{
Análise dos resíduos em modelos de regressão não linear ajustados aos dados de crotalária juncea por diferentes métodos de estimação
}

\author{
Residual analysis in nonlinear regression models adjusted to sunn hemp \\ data by different estimation methods
}

\section{Cláudia Marques de Bem', Luciane Flores Jacobi", Alberto Cargnelutti Filho'II}

\begin{abstract}
RESUMO
O objetivo deste trabalho foi verificar a qualidade dos resíduos nos modelos de regressão não linear Gompertz e Logístico estimados por diferentes métodos. Foram avaliados os caracteres da cultura de crotalária juncea: da massa de matéria fresca (folha, caule, raiz, parte aérea e total) e da massa de matéria seca (folha, caule, raiz, parte aérea e total) em função dos dias após a semeadura. Posteriormente, foram analisados os diferentes métodos de estimação dos parâmetros dos modelos: o Método dos Mínimos Quadrados e o Método da Máxima Verossimilhança. Foram verificados os pressupostos dos resíduos dos caracteres, por meio dos seguintes testes: teste de Shapiro-Wilk, teste de Breusch-Pagan e teste de Durbin-Watson. Para estes caracteres produtivos, foram ajustados os modelos Gompertz e Logístico em função dos dias após a semeadura. Pode-se concluir que os métodos de estimativa dos parâmetros dos modelos de regressão não linear (Método dos Mínimos Quadrados e Método da Máxima Verossimilhança) não diferem quanto a qualidade dos resíduos e nem quanto aos valores dos testes que avaliam o atendimento dos pressupostos.
\end{abstract}

Palavras-chave: Avaliação dos pressupostos. Método dos mínimos quadrados. Método da máxima verossimilhança

\begin{abstract}
The objective of this research was to verify the quality of the residues in the nonlinear regression model Gompertz and Logistic estimated by different parameter. The traits of sunn hemp: fresh matter mass (leaf, stem, root, shoot and total), dry matter mass (leaf, stem, root, shoot, and total) depending on the days after sowing. The different estimation methods for the model parameters were analyzed: the Least Squares Method and the Maximum Likelihood Method The character residual assumptions were verified using the following tests: Shapiro-Wilk test, Breusch-Pagan test and For these productive characters, the Gompertz and Logistic models were adjusted as a function of the days after sowing. It can be concluded that the parameter estimation methods of the nonlinear regression models (Least Squares Method and Maximum Likelihood) do not differ regarding the quality of the residuals or the values of the tests that assess the fulfillment of the assumptions.
\end{abstract}

Keywords: Assessment of assumptions. Least Squares Method. Maximum Likelihood Method

III Universidade Federal de Santa Maria. Brasil. e-mail: alberto.cargnelutti.filho@gmail.com 


\section{INTRODUÇÃO}

Uma forma de estudar o planejamento, execução, coleta dos dados, análise e interpretação dos resultados dos experimentos é por meio da experimentação. Este assunto é importante para todo o profissional ligado direta ou indiretamente à pesquisa. A análise estatística é fundamental na experimentação, uma vez que todo experimento envolve dados que estão sujeitos ao erro experimental. A relação de variáveis pode ser descrita por meio de funções matemáticas, também chamadas de modelos de regressão, sendo que estes modelos se dividem em duas classes: os modelos de regressão linear e os modelos de regressão não linear.

A regressão não linear é uma forma de análise em que os dados são modelados por uma função que é uma combinação não linear de parâmetros dos modelos e depende de uma ou mais variáveis independentes. Os modelos consistem em uma formulação que simula um fenômeno do mundo real, de forma a ser possível fazer previsões, e podem ser utilizados nas mais diversas áreas de pesquisa (FERNANDES, 2012). Na análise de regressão não linear, assim como na regressão linear, a análise dos resíduos de um modelo é feita para verificar a plausibilidade das pressuposições envolvidas de normalidade, homocedasticidade e independência dos erros. O processo de estimação dos parâmetros dos modelos não lineares é complexo, pois, além de escolher o modelo que melhor descreve a variável resposta, a interpretação correta dos parâmetros do modelo se torna importante. A obtenção das estimativas dos parâmetros pode ser realizada pelo Método dos Mínimos Quadrados (MMQ) ou pelo Método da Máxima Verossimilhança (MMV).

Quando são utilizados os modelos não lineares, o sistema de equações normais não é resolvido facilmente, sendo necessários processos iterativos para a obtenção destas estimativas. Contudo, o não atendimento das pressuposições (normalidade, homocedasticidade e independência dos erros) implicará no método de estimativa dos parâmetros dos modelos. Portanto, o estudo mais detalhado sobre as influências do não atendimento dos pressupostos para a análise estatística e verificar se há diferença nos resultados utilizando diferentes métodos de estimação dos parâmetros é de suma importância. 
Diante do exposto, esta pesquisa tem por objetivo verificar a qualidade dos resíduos no modelo de regressão não linear estimados por dois métodos de estimação dos parâmetros.

\section{METODOLOGIA}

Foram utilizados os dados provenientes de dois ensaios de uniformidade com a cultura de crotalária juncea (Crotalaria juncea L.) no ano agrícola de 2014/2015, na área experimental do Departamento de Fitotecnia da Universidade Federal de Santa

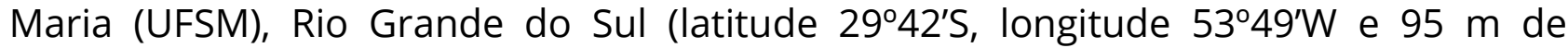
altitude). As sementes de crotalária juncea, foram semeadas em duas épocas de semeadura, sendo a primeira época de semeadura em 22/10/2014 e a segunda época de semeadura em 03/12/2014. A semeadura em ambas as épocas foi realizada em fileiras com 0,5 m entre fileiras e com densidade de 20 plantas por metro de fileira em área útil de $52 \mathrm{~m} \times 50 \mathrm{~m}\left(2.600 \mathrm{~m}^{2}\right)$.

Após a emergência das plântulas de crotalária juncea, para a época 1 a partir dos 7 dias após a semeadura, e para a época 2 a partir dos 13 dias após a semeadura, foram coletadas diariamente e aleatoriamente, quatro plantas. Foram avaliados os caracteres produtivos: massa de matéria fresca de folha (MFF em gramas), massa de matéria fresca de caule (MFC em gramas), massa de matéria fresca de raiz (MFR em gramas), massa de matéria fresca de parte aérea (MFPA = MFF + MFC em gramas), massa de matéria fresca total (MFT em gramas), massa de matéria seca de folha (MSF em gramas), massa de matéria seca de caule (MSC em gramas), massa de matéria seca de raiz (MSR em gramas), massa de matéria seca de parte aérea (MSPA = MSF + MSC em gramas) e massa de matéria seca total (MST em gramas). Para estes caracteres produtivos, foram ajustados os modelos Gompertz e Logístico em função dos dias após a semeadura (DAS) (BEM et al., 2018). Para o modelo de Gompertz foi utilizada a equação: $y_{i}=a \exp \left[-\exp \left(b-c x_{i}\right)\right]$ onde $a$ é o valor assintótico; $b$ é o parâmetro de alocação sem interpretação prática direta, mas importante para manter o formato sigmoidal do modelo, c é o parâmetro associado ao crescimento, valor que indica o índice de precocidade ou maturidade e $x$ é a variável independente (dias após a semeadura). Para o modelo Logístico foi utilizada a expressão: 
$y_{i}=a /\left[1+\exp \left(-b-c x_{i}\right)\right]$ onde $a$ é o valor assintótico; $b$ é o parâmetro de alocação com interpretação prática direta e importante para manter o formato sigmoidal do modelo, c é o parâmetro associado ao crescimento, valor que indica o índice de precocidade ou maturidade e $x$ é a variável independente (dias após a semeadura).

Posteriormente, foram analisados os diferentes métodos de estimação para os parâmetros dos modelos: o Método dos Mínimos Quadrados e Método da Máxima Verossimilhança. O método dos mínimos quadrados tem o princípio que para ajustar uma reta aos valores dos dados, deve-se procurar a reta tal que a soma dos quadrados das distâncias verticais de cada ponto à reta seja a menor possível (HILL et al., 2010). Por meio deste método, tomam-se os quadrados das distâncias para evitar que grandes distâncias positivas sejam canceladas pelas negativas. Foi utilizada a equação: $y=f(x ; \theta)+\varepsilon$, em que $y=\left(\mathrm{y}_{1}, \ldots, \mathrm{y}_{n}\right)^{t} \mathrm{e}^{x}=\left(\mathrm{x}_{1}, \ldots, \mathrm{x}_{n}\right)^{\mathrm{t}}$ são os vetores de variáveis resposta e variável explicativa, respectivamente; $\theta=\left(\theta_{1}, \ldots, \theta_{p}\right)^{t}$ é o vetor de parâmetros desconhecidos. Portanto, $\left.f(x ; \theta)=\mathrm{f}\left(\mathrm{x}_{1} ; \theta\right), \ldots, \mathrm{f}\left(\mathrm{x}_{\mathrm{n}} ; \theta\right)\right)^{\mathrm{t}}$ é uma função das variáveis regressoras e dos parâmetros chamada de função esperança ou função de regressão e $\varepsilon=\left(\varepsilon_{1}, \ldots, \varepsilon_{n}\right)^{t}$ é o vetor dos erros aleatórios. Por consequência de inferências, assume-se que os erros são variáveis aleatórias independentes e identicamente distribuídos, normais com média 0 e variância constante $\sigma^{2} \ln _{n}$, onde $\ln$ representa a matriz identidade de ordem n (MATTOS, 2013).

Para a obtenção das estimativas dos mínimos quadrados, utilizou-se método de Gauss-Newton ou método da linearização. Já para o Método da Máxima Verossimilhança, foi utilizada a função: $\mathrm{L}(f(x ; \theta)$, em que y é a variável e $\theta$ é o parâmetro fixo, e o problema consiste em obter o valor de $\hat{\theta}$ que maximiza essa função. O estimador de máxima verossimilhança $\hat{\theta}$ é o vetor que faz $L(\hat{\theta} ; y)>L(\hat{\bar{\theta}} ; y)$, sendo $\widehat{\hat{\theta}}$ qualquer outro estimador de $\theta$. Foram verificados os pressupostos dos resíduos, por meio dos seguintes testes: teste de Shapiro-Wilk para a normalidade dos resíduos (SHAPIRO e WILK, 1965) dada pela equação 1:

$$
w=\frac{b^{2}}{\sum_{i=1}^{n} x(i)-\bar{x}}
$$


em que $\bar{x}$ é a média amostral; x(i) são os valores amostrais ordenados e o valor b é calculado com base nas médias, variâncias e covariâncias das estatísticas de ordem de uma amostra aleatória de tamanho $\mathrm{n}$ de uma distribuição normal. Valores menores de w indicam evidências sobre os desvios de normalidade.

O teste de Breusch-Pagan para a homocedasticidade dos resíduos (BREUSCH e PAGAN, 1979) dada pela equação 2 :

$$
u i=\frac{e_{i}^{2}}{\frac{S Q E E}{n}}
$$

E o teste de Durbin-Watson para a presença de autocorrelação dos resíduos (DURBIN e WATSON, 1950) dada pela equação 3:

$$
D W=\frac{\left.\sum_{t=2}^{n}\left(\varepsilon_{i}-\varepsilon_{i-1}\right)\right)^{2}}{\sum_{t=1}^{n}\left(\varepsilon_{i}\right)^{2}}
$$

em que $\varepsilon_{\mathrm{i}}$ é o resíduo no tempo i, e $\varepsilon_{\mathrm{i}-1}$ é o resíduo no tempo anterior a i. O valor de DW está entre 0 e 4 . Valores calculados próximos de 0 indicam presença de autocorrelação positiva, próximos de 2 significam que os resíduos são independentes e próximos de 4 indicam presença de autocorrelação negativa.

Para todos os testes foi considerado um nível de significância de 5\%. Para estas análises foi utilizado o software estatístico R (R DEVELOPMENT CORE TEAM, 2019).

\section{Resultados e discussão}

A análise dos pressupostos associados aos modelos ajustados é de suma importância para que os resultados tenham confiabilidade. Primeiramente, a estimação para os parâmetros dos modelos foi realizada por meio do Método dos Mínimos Quadrados (MMQ). 
Observou-se que na primeira época de semeadura, o pressuposto de normalidade dos resíduos não foi satisfeito para os caracteres massa de matéria fresca de folha (MFF) massa de matéria fresca de caule (MFC) no modelo de Gompertz. Já o pressuposto de homocedasticidade não foi atendido no caractere massa de matéria seca de caule (MSC) no modelo Logístico. Entretanto, para época 2 de semeadura o único pressuposto descumprido foi o de homocedasticidade nos caracteres MFF e massa de matéria seca de folha (MSF) ambas no modelo de Gompertz (Tabela 1).

Alguns autores ressaltam a importância da utilização dos MMQ tais como: MORGANO et al. (2007), desenvolvendo uma metodologia analítica não destrutiva e aplicação do método de regressão por mínimos quadrados parciais (PLS); CARVALHO et al. (2009), comparando o método de estimação dos MMQ com o método da tentativa; NETO et al. (2013), ajustando os modelos não lineares a dados de crescimento com estrutura de erros independentes e autoregressivos de primeira ordem; FERNANDES et al. (2014), selecionando os modelos não lineares para a descrição das curvas de crescimento do fruto do cafeeiro; PEREIRA et al. (2014), ajustando a função logística a dados de crescimento; REIS et al. (2014), estudando os modelos de regressão não linear para descrição do crescimento de plantas de alho; e BEM et al. (2018), estudando os modelos Gompertz e Logístico para caracteres produtivos de crotalária juncea em épocas de semeadura. 
Tabela 1 - Valor-p dos testes de Shapiro-Wilk (SW), Breusch-Pagan (BP) e Durbin-Watson (DW) aplicados sobre os resíduos dos modelos de Gompertz e Logístico, para os caracteres produtivos de crotalária juncea, em duas épocas de semeadura utilizando o Método dos Mínimos Quadrados.

\begin{tabular}{|c|c|c|c|c|c|c|c|}
\hline & \multicolumn{3}{|c|}{ Época 1} & \multicolumn{3}{|c|}{ Época 2} \\
\hline & & \multicolumn{3}{|c|}{ (semeadura em 22/10/2014) } & \multicolumn{3}{|c|}{ (semeadura em 03/12/2014) } \\
\hline Caractere* $^{*}$ & Modelo & SW & $\mathrm{BP}$ & DW & SW & $\mathrm{BP}$ & DW \\
\hline \multirow[t]{2}{*}{ MFF } & Gompertz & 0,0475 & 0,1007 & 0,0576 & 0,0817 & 0,0432 & 0,0817 \\
\hline & Logístico & 0,0769 & 0,1510 & 0,0542 & 0,0527 & 0,0501 & 0,0616 \\
\hline \multirow[t]{2}{*}{ MFC } & Gompertz & 0,0415 & 0,0658 & 0,9793 & 0,2983 & 0,2706 & 0,1124 \\
\hline & Logístico & 0,1684 & 0,0500 & 3 & 48 & 0,4048 & 0,1357 \\
\hline \multirow[t]{2}{*}{ MFR } & Gom & 3 & 0 & 9 & & & 7159 \\
\hline & Logístico & 0,1065 & 0,5155 & 0,5005 & 0,1134 & 0,0760 & 0,7496 \\
\hline \multirow[t]{2}{*}{ MFPA } & Gompertz & 0,0589 & 0,1651 & 0,7700 & 0,1745 & 0,0900 & 0,1345 \\
\hline & co & 0,1807 & 0,1393 & 5958 & 08 & 0,1503 & 1236 \\
\hline \multirow[t]{2}{*}{ MFT } & Gompertz & 0,0 & 0,1 & & & & 969 \\
\hline & Logí & 0,0745 & 0,05 & 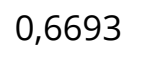 & & 0,1 & 0,1879 \\
\hline \multirow[t]{2}{*}{ MSF } & Gompertz & 0,0879 & 0,1738 & 0,3103 & 0,2449 & 0,0468 & 0,2583 \\
\hline & Logístico & 0,1292 & & & & & 0,3173 \\
\hline \multirow[t]{2}{*}{ MSC } & Gompertz & 0,8002 & 0,8067 & 0,9881 & 0,4266 & 0,3728 & 0,9039 \\
\hline & & 0,8131 & 0,0402 & 0,9874 & 0,3348 & 0,3589 & 0,9124 \\
\hline \multirow[t]{2}{*}{ MSR } & Gompertz & 0,2777 & 0,7099 & 0,16 & 0,1 & 0 , & 0,6076 \\
\hline & Logístico & 0,1042 & 0,4578 & 0,5698 & 0,1602 & 0,1893 & 0,6385 \\
\hline \multirow[t]{2}{*}{ MSPA } & Gompertz & 0,4391 & 0,2132 & 0,9284 & 0,3873 & 0,2884 & 0,8790 \\
\hline & Logístico & 0,0694 & 0,5642 & 0,9184 & 0,0773 & 0,2421 & 0,8545 \\
\hline \multirow[t]{2}{*}{ MST } & Gompertz & 0,2322 & 0,3253 & 0,7270 & 0,4455 & 0,2395 & 0,7089 \\
\hline & Logístico & 0,1481 & 0,3070 & 0,9298 & 0,0544 & 0,1288 & 0,6085 \\
\hline
\end{tabular}

${ }^{*}$ MFF = massa de matéria fresca de folha; MFC = massa de matéria fresca de caule; MFR = massa de matéria fresca de raiz; MFPA = massa de matéria fresca de parte aérea, MFT = massa de matéria fresca total; MSF = massa de matéria seca de folha; $M S C=$ massa de matéria seca de caule; MSR = massa de matéria seca de raiz; MSPA = massa de matéria seca de parte aérea e MST = massa de matéria seca total.

Contudo, devido ao não atendimento destes pressupostos para estes caracteres, foi utilizado o Método da Máxima Verossimilhança (MMV). Observou-se que os resultados foram semelhantes quando comparados com o Método dos Mínimos Quadrados, em que os caracteres MFF e MFC não atenderam ao pressuposto de normalidade e o caractere MSC não atendeu ao pressuposto de homogeneidade na primeira época de semeadura (Tabela 2). Para a segunda época de semeadura, também foi observado comportamento similar ao encontrado para o método dos mínimos quadrados, em que os caracteres MFF não atenderam ao pressuposto de homogeneidade de variância $(p \leq 0,05)$. 
Tabela 2 - Valor-p dos testes de Shapiro-Wilk (SW), Breusch-Pagan (BP) e Durbin-Watson (DW) aplicados sobre os resíduos dos modelos de Gompertz e Logístico, para os caracteres produtivos de crotalária juncea, em duas épocas de semeadura utilizando o método da máxima verossimilhança.

\begin{tabular}{|c|c|c|c|c|c|c|c|}
\hline \multirow[b]{2}{*}{ Caractere* } & \multirow[b]{2}{*}{ Modelo } & \multicolumn{3}{|c|}{$\begin{array}{c}\text { Época } 1 \\
\text { (semeadura em 22/10/2014) }\end{array}$} & \multicolumn{3}{|c|}{$\begin{array}{c}\text { Época } 2 \\
\text { (semeadura em 03/12/2014) }\end{array}$} \\
\hline & & SW & $\mathrm{BP}$ & DW & SW & $\mathrm{BP}$ & DW \\
\hline \multirow[t]{2}{*}{ MFF } & Gompertz & 0,0469 & 0,1010 & 0,0579 & 0,0818 & 0,0433 & 0,0819 \\
\hline & Logístico & 0,0770 & 0,1511 & 0,0541 & 0,0528 & 0,0501 & 0,0616 \\
\hline \multirow[t]{2}{*}{ MFC } & Gompertz & 0,0413 & 0,0655 & 0,9792 & 0,2985 & 0,2708 & 0,1127 \\
\hline & Logístico & 0,1686 & 0,0502 & 0,9461 & 0,0650 & 0,4049 & 0,1359 \\
\hline \multirow[t]{2}{*}{ MFR } & Gompertz & 0,0962 & 0,3958 & 0,5388 & 0,0621 & 0,0655 & 0,7158 \\
\hline & Logístico & 0,1064 & 0,5153 & 0,5006 & 0,1136 & 0,0763 & 0,7499 \\
\hline \multirow[t]{2}{*}{ MFPA } & Gompertz & 0,0589 & 0,1651 & 0,7700 & 0,1746 & 0,0902 & 0,1347 \\
\hline & Logístico & 0,1809 & 0,1393 & 0,5958 & 0,0509 & 0,1506 & 0,1235 \\
\hline \multirow[t]{2}{*}{ MFT } & Gompertz & 0,0502 & 0,1088 & 0,8196 & 0,2319 & 0,1223 & 0,1963 \\
\hline & Logístico & 0,0742 & 0,0966 & 0,6692 & 0,0545 & 0,1904 & 0,1881 \\
\hline \multirow[t]{2}{*}{ MSF } & Gompertz & 0,0879 & 0,1737 & 0,3107 & 0,2449 & 0,0466 & 0,2588 \\
\hline & Logístico & 0,1294 & 0,1723 & 0,2319 & 0,2293 & 0,0509 & 0,3178 \\
\hline \multirow[t]{2}{*}{ MSC } & Gompertz & 0,8004 & 0,8069 & 0,9884 & 0,4265 & 0,3727 & 0,9038 \\
\hline & Logístico & 0,8133 & 0,0401 & 0,9875 & 0,3346 & 0,3582 & 0,9126 \\
\hline \multirow[t]{2}{*}{ MSR } & Gompertz & 0,2778 & 0,7000 & 0,1626 & 0,1792 & 0,1572 & 0,6078 \\
\hline & Logístico & 0,1045 & 0,4579 & 0,5699 & 0,1604 & 0,1895 & 0,6384 \\
\hline \multirow[t]{2}{*}{ MSPA } & Gompertz & 0,4393 & 0,2135 & 0,9286 & 0,3877 & 0,2886 & 0,8792 \\
\hline & Logístico & 0,0697 & 0,5643 & 0,9185 & 0,0775 & 0,2423 & 0,8547 \\
\hline \multirow[t]{2}{*}{ MST } & Gompertz & 0,2324 & 0,3253 & 0,7273 & 0,4450 & 0,2393 & 0,7091 \\
\hline & Logístico & 0,1483 & 0,3072 & 0,9297 & 0,0546 & 0,1286 & 0,6087 \\
\hline
\end{tabular}

${ }^{*}$ MFF = massa de matéria fresca de folha; MFC = massa de matéria fresca de caule; MFR = massa de matéria fresca de raiz; MFPA = massa de matéria fresca de parte aérea, MFT = massa de matéria fresca total; MSF = massa de matéria seca de folha; $M S C=$ massa de matéria seca de caule; $M S R=$ massa de matéria seca de raiz; MSPA = massa de matéria seca de parte aérea e MST = massa de matéria seca total.

Estes resultados podem ser explicados por meio de alguns pontos importantes. Primeiramente, ressalta-se que tanto para o MMQ quanto para o MMV utilizaram o mesmo conjunto de dados com os mesmos números de observações, sendo que para primeira época de semeadura foram 94 dias de avaliação (período entre 7 e 100 DAS) e para a segunda época de semeadura 76 dias de avaliação (período entre 13 e 88 DAS). Observa-se, que as variáveis respostas $y_{i}$ foram as mesmas em ambos os métodos e também a variável independente $\left(x_{i}\right)$ que nesta presente pesquisa se utilizou dias após a semeadura (DAS). Contudo, estas são suposições que podem explicar a semelhança dos resultados encontrados para ambos os métodos. Contudo, devem-se considerar alguns aspectos sobre os métodos utilizados considerando este 
conjunto de dados. Estes métodos constituem duas abordagens comumente utilizadas para estimar os parâmetros das populações utilizando-se uma amostra aleatória. As estimativas de mínimos quadrados são calculadas ajustando uma linha de regressão aos pontos de um conjunto de dados com a soma mínima dos desvios elevados ao quadrado (HILL, JUDGE, GRIFFITHS, 2010). Já a função de verossimilhança indica quão provável a amostra observada é como uma função de possíveis valores de parâmetro. Contudo, maximizar a função de verossimilhança determina os parâmetros que tem maior probabilidade de produzir os dados observados. Estatisticamente, o MMV é recomendado para amostras grandes, pois este método é versátil, aplicável à maioria dos modelos e a diferentes tipos de dados, produzindo estimadores de menor variância, porém envolve equações em geral não lineares e implícitas. Portanto, observa-se que pelo conjunto de dados da presente pesquisa que o método de estimação dos mínimos quadrados é o mais adequado.

Observou-se que os modelos ajustados foram os modelos de regressão não lineares Gompertz e Logístico e estes possuem a característica de ajustar curvas sigmoides como pode ser visto em BEM et al. (2018). Para estes dados o MMQ, devido a suas características, obteve bons resultados para os caracteres estudados, contudo a utilização do MMV não obteve resultados diferentes dos encontrados pelo MMQ. Por meio da utilização destes dois métodos de estimação dos parâmetros obteve-se o ajuste dos modelos de Gompertz e Logístico nas duas épocas de semeadura. Observa-se nos gráficos o ajuste dos modelos não lineares Gompertz e Logístico (Figuras 1 e 2) utilizando o MMQ, os pontos observados e a curva ajustada para cada caractere. Nota-se a equação para cada modelo em que estão representados os valores dos parâmetros $a, b$ e c e para avaliar a qualidade de ajuste do modelo é utilizado o coeficiente de determinação $\left(R^{2}\right)$. Estas equações são importantes para visualizar o crescimento da planta, e para cada caractere estudado o comportamento é diferente devido aos valores dos parâmetros. Observa-se que os valores do $\mathrm{R}^{2}$ são satisfatórios em todos os caracteres estudados para a primeira época de semeadura. 
Figura 1. Modelo Gompertz (coluna da esquerda) e Logístico (coluna da direita), para os caracteres produtivos de massa de matéria fresca de folha (MFF), massa de matéria fresca de caule (MFC), massa de matéria fresca de raiz (MFR), massa de matéria fresca de parte aérea (MFPA), massa de matéria fresca total (MFT), massa de matéria seca de folha (MSF), massa de matéria seca de caule (MSC), massa de matéria seca de raiz (MSR), massa de matéria seca de parte aérea (MSPA) e massa de matéria seca total (MST), para crotalária juncea na primeira época de semeadura (semeada em 22/10/2014).
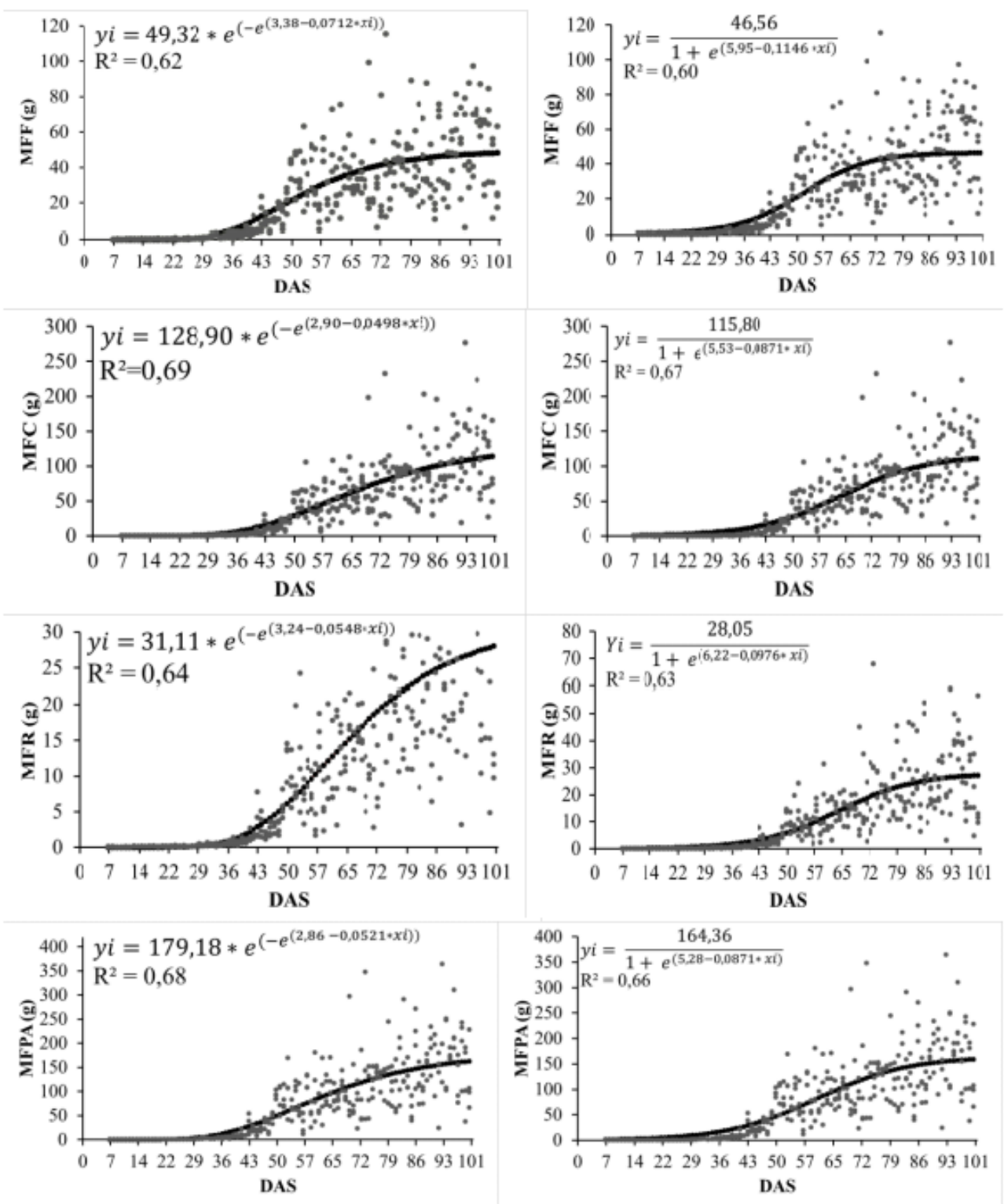

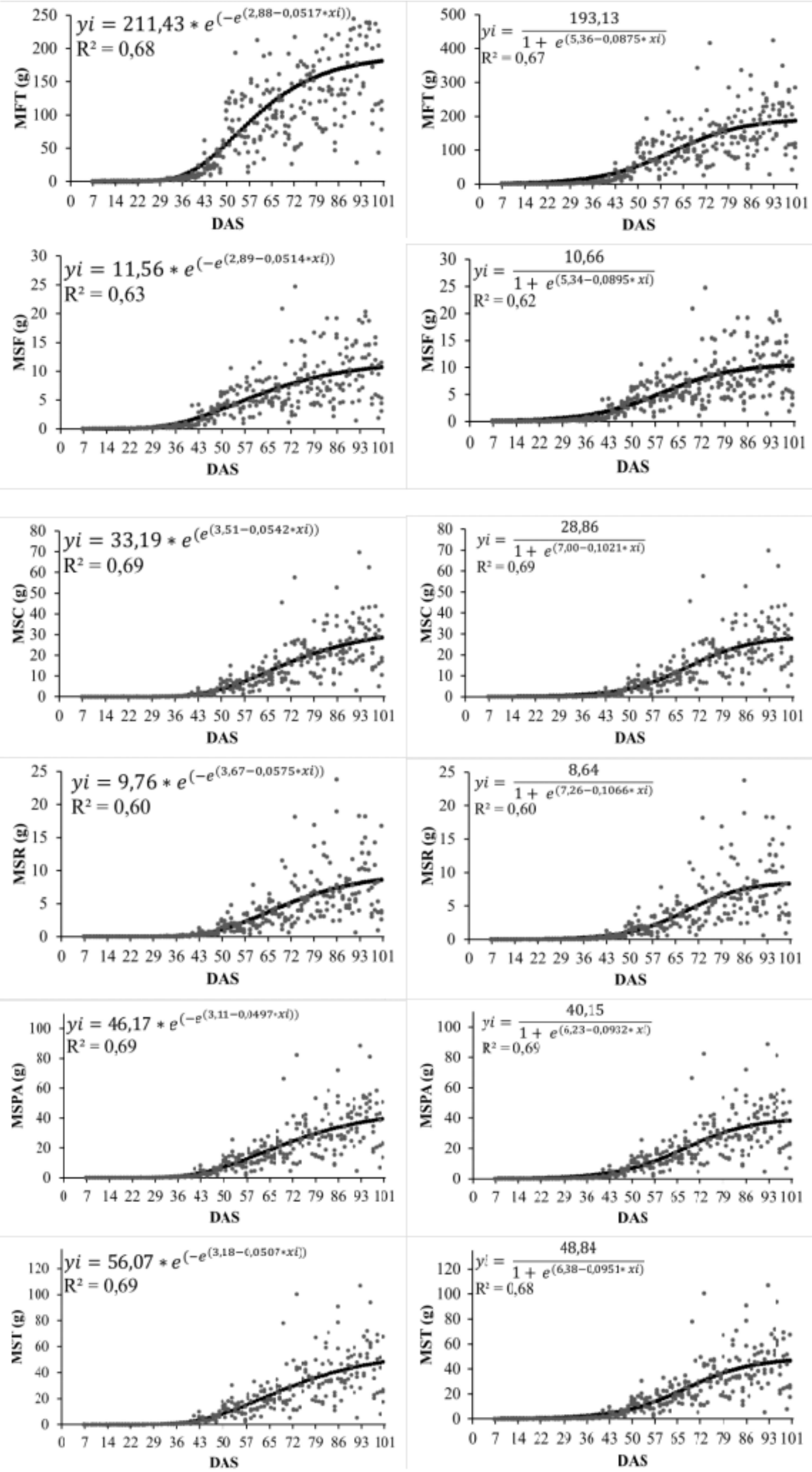
Contudo, para a segunda época de semeadura o $R^{2}$, não foi satisfatório ficando aquém do esperado sendo que o modelo de Gompertz e Logístico não se ajustaram aos dados da cultura de crotalária juncea.

Figura 2. Modelo Gompertz (coluna da esquerda) e Logístico (coluna da direita), para os caracteres produtivos de massa de matéria fresca de folha (MFF), massa de matéria fresca de caule (MFC), massa de matéria fresca de raiz (MFR), massa de matéria fresca de parte aérea (MFPA), massa de matéria fresca total (MFT), massa de matéria seca de folha (MSF), massa de matéria seca de caule (MSC), massa de matéria seca de raiz (MSR), massa de matéria seca de parte aérea (MSPA) e massa de matéria seca total (MST), para crotalária juncea na segunda época de semeadura (semeada em 03/12/2014).
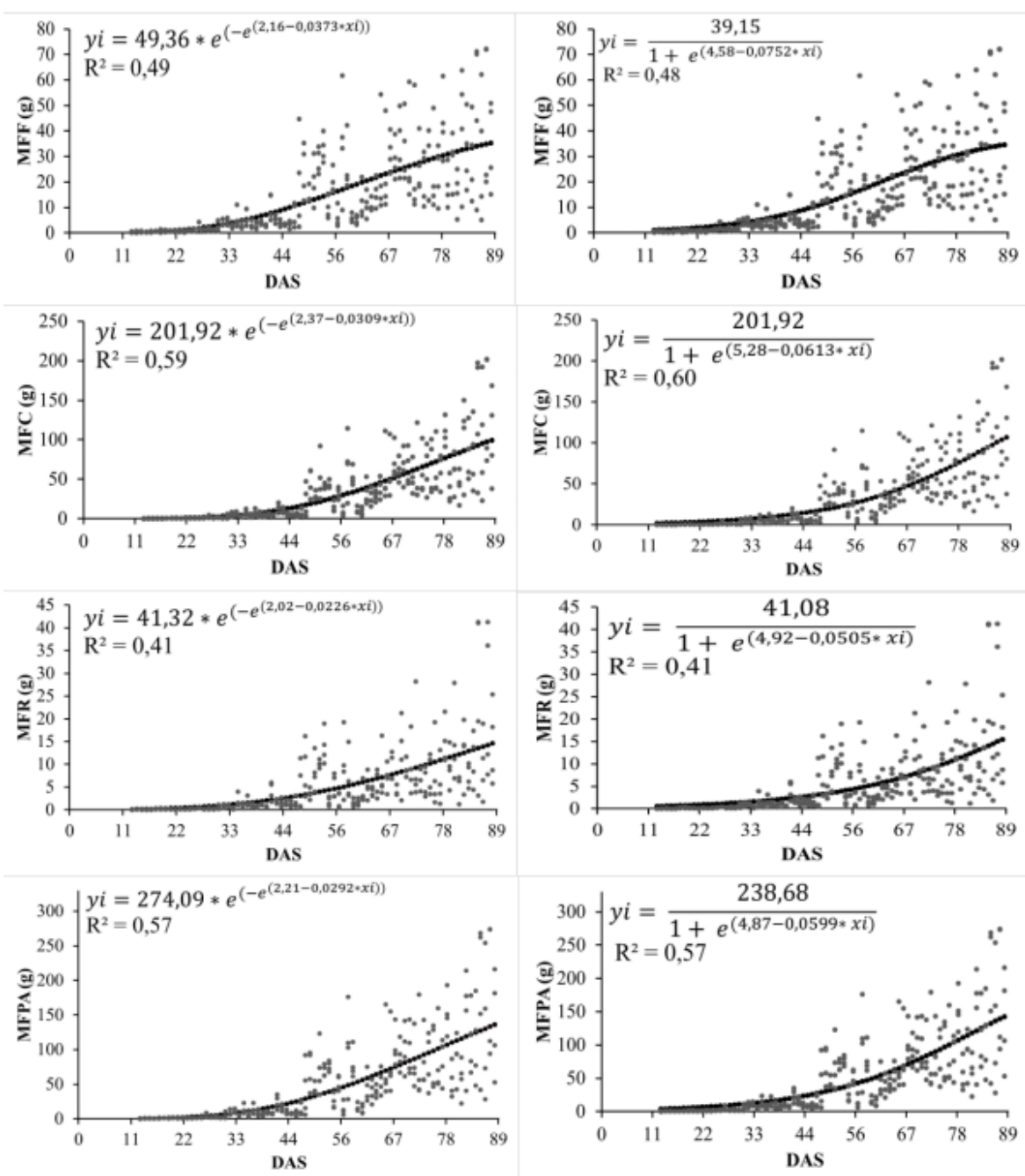

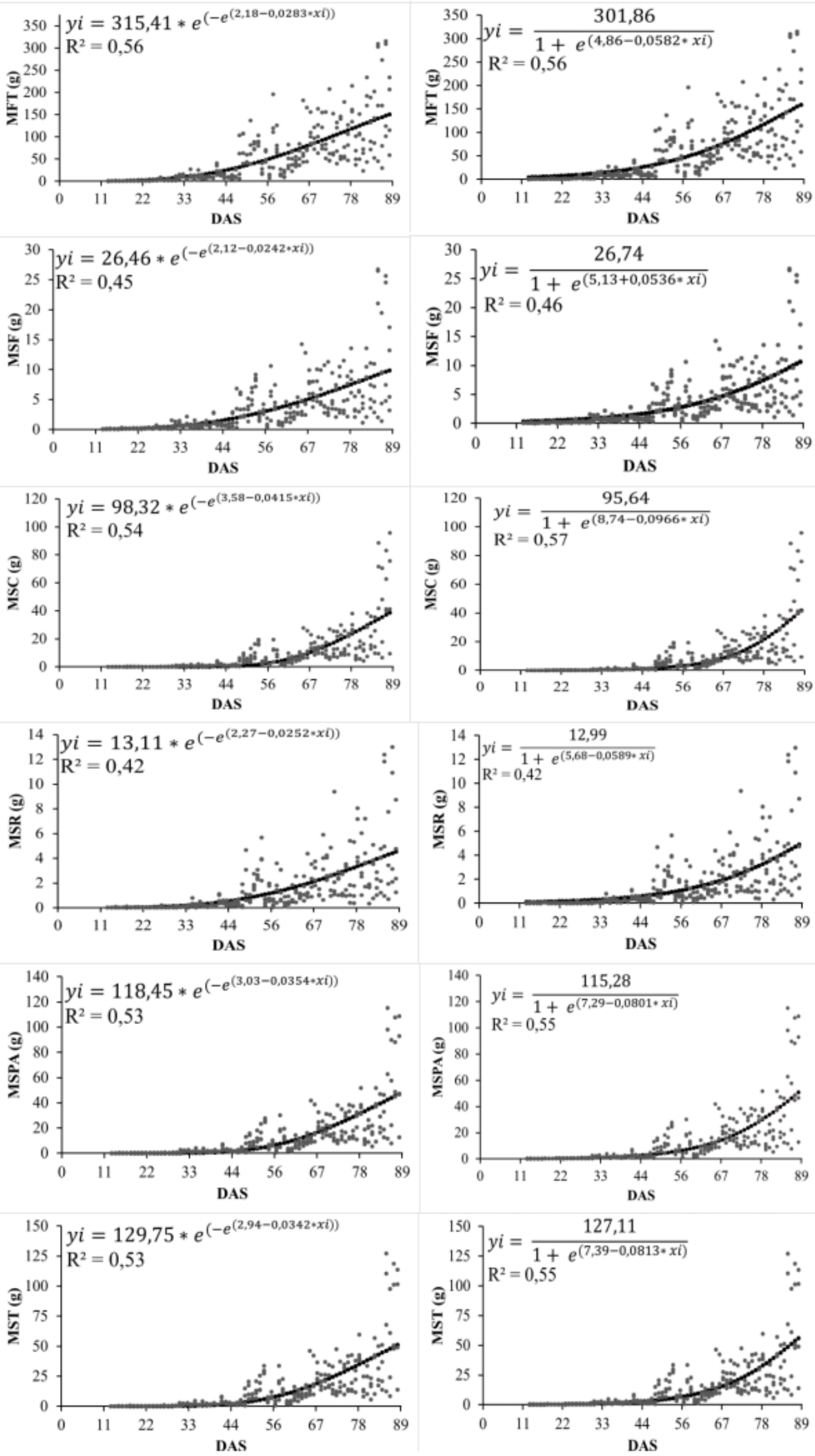
Os mesmos resultados foram encontrados quando se utilizou do MMV, em que os dados utilizados foram os mesmos. Ressalta-se que o MMV não apresentou resultados satisfatórios para os caracteres produtivos da cultura de crotalária juncea.

\section{CONCLUSÕES}

Os Métodos dos Mínimos Quadrados e da Máxima Verossimilhança, utilizados para estimar os parâmetros dos modelos de regressão não linear, não diferem quanto a qualidade dos seus resíduos e nem quanto aos valores dos testes que avaliam o atendimento dos pressupostos. 


\section{REFERÊNCIAS}

BEM CM de, CARGNELUTTI FILHO A, CHAVES GG, KLEINPAUL JA, PEZZINI RV, LAVEZO A. Gompertz and Logistic models to the productive traits of sunn hemp. Journal of Agricultural Science, 2018; 10(1): 225-238.

BREUSCH T, PAGAN A. Teste simples para heterocedasticidade e coeficiente de variação aleatória econométrica. Sociedade Econométrica. 1979; 47: 1287-1294.

CARVALHO RPJ de, VIEIRA SR, GREGO CR. Comparação de métodos para ajuste de modelos de semivariograma da precipitação pluvial anual. Revista Brasileira de Engenharia Agrícola e Ambiental. 2009; 13(4): 443-448.

DURBIN J, WATSON GS. Teste de correlação serial em Mínimos Quadrados de Regressão I. Biometrika. 1950; 37: p.409-428.

FERNANDES TJ, PEREIRA AA, MUNIZ JA, SAVIAN TV. Seleção de modelos não lineares para descrição das curvas de crescimento do fruto do cafeeiro. Coffee Science. 2014; 9(2): 207-215.

FERNANDES TJ. Curva de crescimento do fruto do cafeeiro em diferentes alinhamentos de plantio utilizando modelos não lineares. Lavras, 2012. 80p. Dissertação (Mestrado). Universidade Federal de Lavras, 2012.

HILL RC, JUDGE GG, GRIFFITHS WE. Econometria / R. Carter Hill, William E. Griffiths, George G. Judge. 3.ed.- São Paulo: Saraiva, 2010.

MATTOS TB. Modelos não lineares e suas aplicações. Juiz de Fora. 58p. Monografia. Universidade de Juiz de Fora, 2013.

MORGANO MA, FARIA CG de, FERRÃO MF, FERREIRA, MMC. Determinação de açúcar total em café cru por espectroscopia no infravermelho próximo por regressão por mínimos quadrados parciais. Química Nova. 2007; 30(2): 346-350.

NETO FS, CARVALHO LR de, MISCHAN MM. Ajuste dos modelos não lineares a dados de crescimento com estrutura de erros independentes e autoregressivos de primeira ordem-aplicações. Revista Brasileira de Biometria. 2013; 31(4): 631-644.

PEREIRA GMS. Ajuste da função logística a dados de crescimento. Revista de Estatística. 2014; 3: 35-39.

R DEVELOPMENT CORE TEAM.R: a language and environment for statistical computing. Vienna: R Foundation for Statistical Computing, 2019. 
REIS RM, CECON PR, PUIATTI M, FINGER FL, NASCIMENTO M, SILVA FF, CARNEIRO APS, SILVA AR. Modelos de regressão não linear aplicados a grupos de acesso de alho. Horticultura Brasileira. 2014; 32: 178-183.

SHAPIRO SS, WILK MB. An analysis of variance test for normality. Biometrika. 1965; 52 